Acta Crystallographica Section F

Structural Biology

and Crystallization

Communications

ISSN 1744-3091

Charles E. Nichols, ${ }^{\text {a }}$ Sarah

Sainsbury, ${ }^{\text {b Nick S. Berrow, }}$ David Alderton, ${ }^{\text {b Nigel J. }}$

Saunders, ${ }^{c}$ David K. Stammers ${ }^{\mathrm{a}, \mathrm{b}}$ and Raymond J. Owens ${ }^{\text {b* }}$

a Division of Structural Biology, Henry Wellcome Building for Genomic Medicine, University of Oxford, Roosevelt Drive, Oxford OX3 7BN, England, ' ${ }^{\mathrm{b}}$ The Oxford Protein Production Facility, Henry Wellcome Building for Genomic Medicine, University of Oxford, Roosevelt Drive, Oxford OX3 7BN, England, and ${ }^{\mathrm{c}}$ The Bacterial Pathogenesis and Functional Genomics Group, The Sir William Dunn School of Pathology, University of Oxford, South Parks Road, Oxford OX1 3RE, England

Correspondence e-mail: ray@strubi.ox.ac.uk

Received 24 January 2006

Accepted 27 April 2006

PDB Reference: $\mathrm{P}_{\| \mathrm{II}}$ signal transduction protein, 2gw8, r2gw8sf.

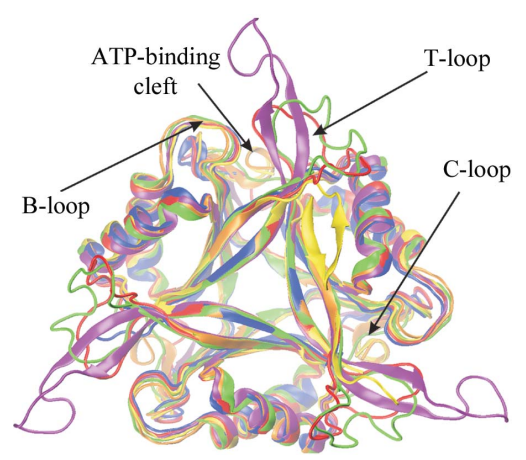

C) 2006 International Union of Crystallography All rights reserved

\section{Structure of the $\mathbf{P}_{\mathrm{II}}$ signal transduction protein of Neisseria meningitidis at $1.85 \AA$ resolution}

The $\mathrm{P}_{\mathrm{II}}$ signal transduction proteins $\mathrm{G} \ln \mathrm{B}$ and $\mathrm{G} \operatorname{lnK}$ are implicated in the regulation of nitrogen assimilation in Escherichia coli and other enteric bacteria. $\mathrm{P}_{\mathrm{II}}$-like proteins are widely distributed in bacteria, archaea and plants. In contrast to other bacteria, Neisseria are limited to a single $\mathrm{P}_{\mathrm{II}}$ protein (NMB 1995), which shows a high level of sequence identity to GlnB and $G \ln K$ from Escherichia coli (73 and 62\%, respectively). The structure of the $\mathrm{P}_{\mathrm{II}}$ protein from $N$. meningitidis (serotype $\mathrm{B}$ ) has been solved by molecular replacement to a resolution of $1.85 \AA$. Comparison of the structure with those of other $\mathrm{P}_{\mathrm{II}}$ proteins shows that the overall fold is tightly conserved across the whole population of related proteins, in particular the positions of the residues implicated in ATP binding. It is proposed that the Neisseria $\mathrm{P}_{\text {II }}$ protein shares functions with $\mathrm{G} \ln \mathrm{B} / \mathrm{G} \operatorname{lnK}$ of enteric bacteria.

\section{Introduction}

The signal transduction protein $\mathrm{P}_{\mathrm{II}}(\mathrm{G} \ln \mathrm{B})$ is best known for its role in the regulation of nitrogen assimilation in enteric bacteria. However, closely related proteins are found in a wide variety of organisms including bacteria, archaea and plants (Arcondeguy et al., 2001; Ninfa \& Jiang, 2005; Ninfa \& Atkinson, 2000). In Escherichia coli, $\mathrm{P}_{\mathrm{II}}$ and its paralogue $\mathrm{GlnK}$, which is only induced under nitrogen limitation, are involved in two signalling pathways that regulate the activity of glutamine synthetase. Firstly, the bifunctional uridylyltransferase/uridylyl-removing enzyme (UTase/UR, the product of $g \ln D$ ) uridylates a conserved tyrosine (Tyr51) of $\mathrm{P}_{\mathrm{II}}$ under nitrogenstarvation conditions, whereas under circumstances of nitrogen excess the enzyme removes UMP from $\mathrm{P}_{\mathrm{II}}$ (Jaggi et al., 1996; Jiang et al., 1998a). Native $\mathrm{P}_{\mathrm{II}}$ activates the adenylation activity of ATase, which in turn inhibits glutamine synthetase, whilst UMP- $\mathrm{P}_{\text {II }}$ reverses this effect (Jaggi et al., 1997; Mangum et al., 1973). Secondly, $\mathrm{P}_{\mathrm{II}}$ affects glutamine synthetase transcription by modulating the activity of the kinase/phosphatase NRII (the ntrB gene product), which controls the phosphorylation status of the transcription factor NRI (the $n t r C$ gene product). In its phosphorylated form, NRI stimulates transcription of the glutamine synthetase gene, whilst the unphosphorylated NRI acts as a repressor. Native $P_{I I}$ binds to NRII, preventing phosphorylation of NRI and hence the activation of GS transcription (Liu \& Magasanik, 1995). UMP-P $\mathrm{P}_{\mathrm{II}}$, which is generated under nitrogen-limiting conditions through uridylation of the T-loop, which extends beyond an otherwise compact trimeric structure, does not bind NRII and hence activation of GS via phosphorylated NRI can proceed (Jiang et al., 1998b). In addition, E. coli $\mathrm{P}_{\mathrm{II}}$ binds cooperatively to two effectors, ATP and $\alpha$-ketogluturate (Jiang et al., $1998 a$ ). The binding of $\alpha$-ketogluturate regulates the interaction between $\mathrm{P}_{\mathrm{II}}$ and ATase, thus integrating signals from carbon and nitrogen metabolism.

Although in E. coli the $\mathrm{P}_{\mathrm{II}}$ paralogue GlnK (van Heeswijk et al., 1995 , 1996) appears to have similar properties to $P_{I I}$ and can form heterotrimers with $\mathrm{P}_{\mathrm{II}}$ (van Heeswijk et al., 2000), GlnK also has distinct roles. In many bacteria, including E. coli, GlnK is associated with regulation of the $a m t B$ gene, which encodes the ammonium transporter (Coutts et al., 2002). GlnK is also involved in the NifLNifA regulatory system of nitrogen-fixing bacteria (Little et al., 2000). 
The X-ray crystal structures of both $\mathrm{P}_{\mathrm{II}}$ (Cheah et al., 1994; Xu et al., 2001) and GlnK (Xu et al., 1998) from E. coli, as well as a number of other $\mathrm{P}_{\mathrm{II}}$ proteins [those from Herbaspirillum seropedicae (Machado Benelli et al., 2002), Synechoccus sp. PCC7942 and Synchocystis sp. PCC6803 (Xu et al., 2003), Thermus thermophilus (Sakai et al., 2005) and Thermotoga maritima (Schwarzenbacher et al., 2004)], have been solved. All share a highly conserved monomer structure arranged into a tightly associated trimer. A key feature of the structures is the so-called T-loop which contains the regulatory uridylylation site (Tyr51) or, in the case of cyanobacteria, a phosphorylation site (Ser49; Forchhammer \& Tandeau de Marsac, 1995). The T-loop has been implicated in the protein-protein interactions of $\mathrm{P}_{\mathrm{II}}$ in E. coli (Jiang, Zucker \& Ninfa, 1997; van Heeswijk et al., 2000; MartinezArgudo \& Contreras, 2002) and shows differences in conformation in the crystal structures.

Neisseria spp. are Gram-negative $\beta$-protobacteria which include many species found only in humans, including successful pathogens. In recent years, the genomes of $N$. meningitidis serotypes A (strain Z2491; Parkhill et al., 2000) and B (strain MC58; Tettelin et al., 2000) and N. gonorrhoeae (strain FA1090; currently unpublished work) have been sequenced and annotated. Each contain a single $\mathrm{P}_{\text {II }}$ protein encoded by a monocistronic operon (NMB1955 in N. meningitidis strain MC58). The three neisserial $\mathrm{P}_{\mathrm{II}}$ proteins have $98 \%$ identical sequences and share $73 \%$ identity to the $\mathrm{P}_{\text {II }}$ from E. coli. As part of a structural proteomics approach to the study of Neisseria, we have solved the structure of $\mathrm{P}_{\mathrm{II}}$ from $N$. meninigitidis (gene locus NMB1995) to $1.85 \AA$ resolution using the semi-automated pipeline of the Oxford Protein Production Facility (OPPF).

\section{Materials and methods}

Cloning, expression and protein purification followed standard OPPF pipeline protocols, as described previously (Ren et al., 2005). Briefly, the $\mathrm{P}_{\mathrm{II}}$ gene (NMB 1955) was amplified from genomic DNA by PCR with the forward primer ggggacaagtttgtacaaaaaagcaggcttcctggaagttctgttccagggeccgATGAAAAAAATCGAGGCGATTGTC and the reverse primer ggggaccactttgtacaagaaagctgggtctcaTCAGACTGCCGCGTCCGAAC incorporating an N-terminal His tag followed by a 3C protease-cleavage site and inserted into the expression vector pDEST17 using Gateway recombinatorial cloning (Invitrogen). Expression was induced by the addition of $0.5 \mathrm{mM}$ IPTG and the protein was purified by a combination of Ni-NTA affinity chromatography and gel filtration. The $\mathrm{N}$-terminal His tag was removed by cleavage with $3 \mathrm{C}$ protease prior to gel filtration. The protein was crystallized using the nanodrop crystallization procedure with standard OPPF protocols (Walter et al., 2003). Hits from this initial screening exercise were then tested in-house using a MAR 345

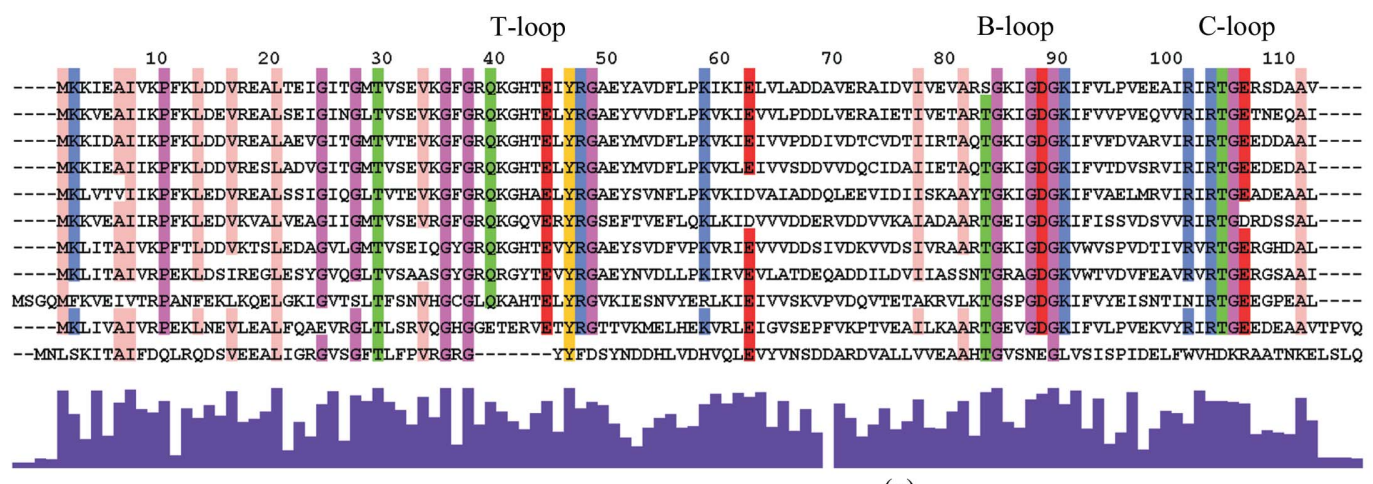

(a)

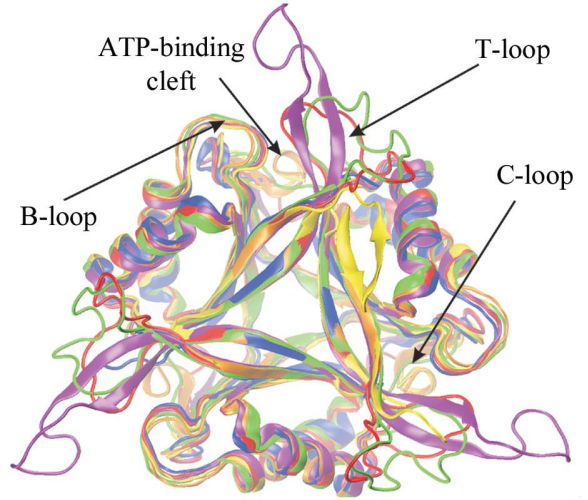

(b)

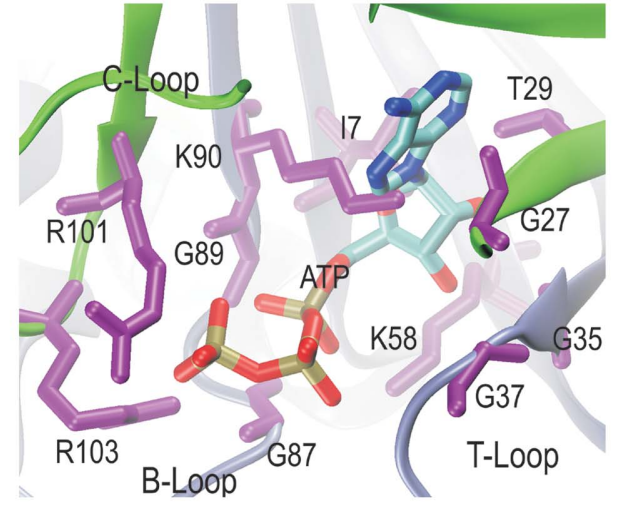

(c)

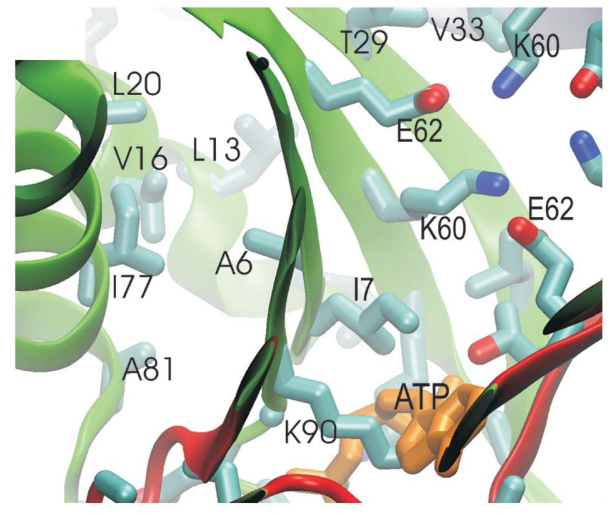

(d)

Figure 1

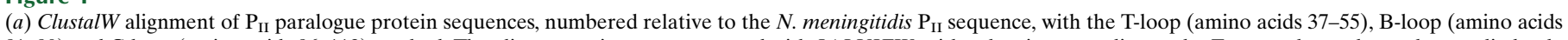

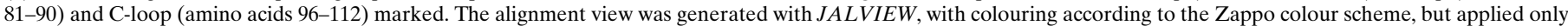

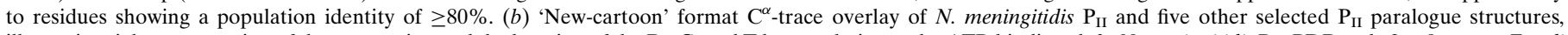

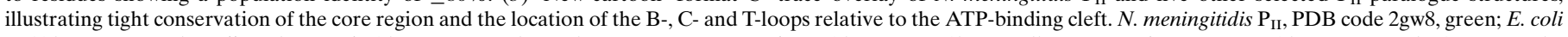

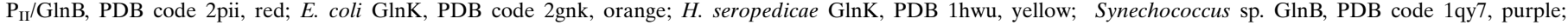

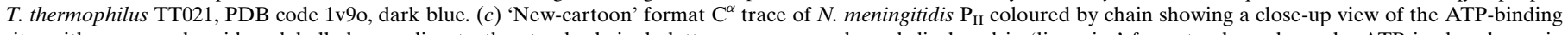

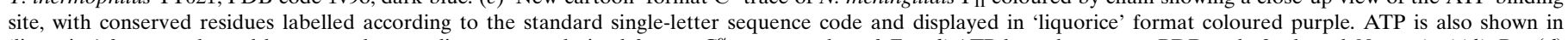

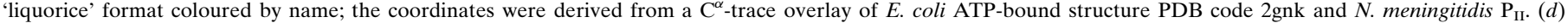

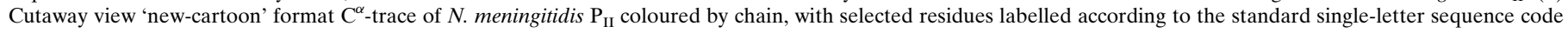

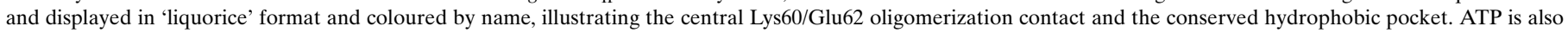
shown in 'liquorice' format coloured orange, with coordinates derived from a $\mathrm{C}^{\alpha}$-trace overlay of $E$. coli ATP-bound structure PDB code $2 \mathrm{gnk}$ and $N$. meningitidis $\mathrm{P}_{\mathrm{II}}$. 
Table 1

Data-collection and processing statistics.

Values in parentheses are for outer shell data.

\begin{tabular}{|c|c|}
\hline Space group & $P 6_{3}$ \\
\hline Unit-cell parameters $\left(\AA,^{\circ}\right)$ & $\begin{array}{c}a=b=61.14, c=48.07 \\
\alpha=\beta=90, \gamma=120\end{array}$ \\
\hline Resolution range & $30.00-1.85(1.92-1.85)$ \\
\hline Redundancy & $5.5(5.4)$ \\
\hline Completeness $(\%)$ & $99.8(100.0)$ \\
\hline$R_{\text {merge }} \dagger$ & $0.065(0.418)$ \\
\hline$I / \sigma(I)$ & $28.1(4.7)$ \\
\hline No. of subunits in ASU & 1 \\
\hline$R_{\text {work }}$ 末 $(\%)$ & 17.7 \\
\hline$R_{\text {free丰 }}(\%)$ & 21.9 \\
\hline Residues in most favoured regions§ (\%) & 95.2 \\
\hline Residues in additionally allowed regions§ (\%) & 4.8 \\
\hline \multicolumn{2}{|l|}{ Mean $B$ factors } \\
\hline All atoms & 34.0 \\
\hline \multicolumn{2}{|l|}{ Protein } \\
\hline Main chain & 22.7 \\
\hline Side chain & 30.5 \\
\hline Water & 55.6 \\
\hline R.m.s.d bond lengths $(\AA)$ & 0.005 \\
\hline R.m.s.d. bond angles $\left({ }^{\circ}\right)$ & 1.24 \\
\hline
\end{tabular}

$\dagger R_{\text {merge }}=\sum\left|I_{\mathrm{obs}}-\langle I\rangle\right| / \sum\langle I\rangle . \quad \neq R=\sum_{h k l}\left|F_{\mathrm{o}}(h k l)-F_{\mathrm{c}}(h k l)\right| / \sum_{h k l}\left|F_{\mathrm{o}}(h k l)\right|$.

$\S$ Ramachandran plot results from PROCHECK.

image-plate system on a Rigaku generator equipped with a $\mathrm{Cu}$ anode and Osmic multilayer optics, giving $\mathrm{Cu} K \alpha$ radiation with $\lambda=1.5418 \AA$. The best diffraction observed $\left(d_{\text {min }}<2.0 \AA\right)$ was from a $75 \times 25 \times 25 \mu \mathrm{m}$ rod-shaped crystal grown in Hampton Cryo Screen I condition No. 31 [0.17 $M$ ammonium sulfate, 25.5\% PEG 4000, $15 \%(v / v)$ glycerol]. This crystal was therefore frozen and dry-shipped to Daresbury for data collection. Indexing, integration and merging of data images were carried out with DENZO and SCALEPACK (Otwinowski \& Minor, 1997). Rotation-function searches, translation searches and initial rigid-body Patterson correlation refinement were carried out using CNS (Brünger et al., 1998) and molecularreplacement solutions were checked by displaying the transformed coordinates in $O$, as described in Jones et al. (1991). Rigid-body, positional and $B$-factor refinement, simulated annealing and initial water picking were carried out in CNS. Manual rebuilding, including insertion of ions, ligands and extra water molecules, was carried out using the program $O$. Homologous sequences to $N$. meningitidis $\mathrm{P}_{\mathrm{II}}$ were selected from the Integrated Microbial Genomes (IMG) database (http://img.jgi.doe.gov/cgi-bin/pub/main.cgi), aligned with ClustalW (Thompson et al., 1994; Chenna et al., 2003) and visualized using JALVIEW (Clamp et al., 2004). Model quality was assessed using PROCHECK (Laskowski et al., 1993). The final N. meningitidis $\mathrm{P}_{\mathrm{II}}$ model was overlaid with the previously released $\mathrm{G} \operatorname{lnB}$ and $\mathrm{G} \ln \mathrm{K}$ structures using TOPP (Collaborative Computational Project, Number 4, 1994) and the results were compared visually in $O$ and $V M D$ (Humphrey et al., 1996). Final figures were prepared from VMD screenshots using Corel11.

\section{Results and discussion}

The structure of $\mathrm{P}_{\text {II }}$ from $N$. meningitidis was determined by molecular replacement using the $\mathrm{PDB}$ model 1 pil $\left(\right.$ E. coli $\left.\mathrm{P}_{\mathrm{II}}\right)$ to a resolution of $1.85 \AA$ (Table 1 ). As with the E. coli crystal structure, $\mathrm{P}_{\mathrm{II}}$ from $N$. meningitidis has a single molecule in the asymmetric unit. Each molecule of the trimer (the normal biological oligomeric state) is therefore in a crystallographically equivalent environment, indicating that all three chains have the same conformational state. The flexible T-loop (residues 37-55) is semi-disordered from residues 38 to 52 in the $N$. meningitidis $\mathrm{P}_{\mathrm{II}}$ crystal structure, although sufficient residual density was still visible in low-contoured difference maps $\left(2.2 \sigma F_{\mathrm{o}}-F_{\mathrm{c}}\right.$ density) to allow an approximate fit based on a rigidbody overlay of the T-loop from E. coli $\mathrm{P}_{\mathrm{II}}$. This section is thus included in Figs. 1(b), 1(c) and 1(d) for comparative purposes, but is omitted from the final deposited coordinates (PDB code 2gw8).

Comparing $\mathrm{C}^{\alpha}$-trace overlays of $N$. meningitidis $\mathrm{P}_{\mathrm{II}}$ with five additional $\mathrm{P}_{\mathrm{II}}$ structures obtained from the PDB, the mean $\mathrm{C}^{\alpha}$ r.m.s.d. for the core section, excluding the flexible T-loop, is just $0.9 \AA$, with a range of $0.7-1.0 \AA$ (Fig. $1 b ; N$. meningitidis $\mathrm{P}_{\mathrm{II}}$ is in green, E. coli $\mathrm{P}_{\mathrm{II}} / \mathrm{GlnB}$ is in red, E. coli $\mathrm{G} \operatorname{lnK}$ is in orange, H. seropedicae $\mathrm{GlnK}$ is in yellow, Synechococcus sp. GlnB is in purple and T. thermophilus TT021 is in dark blue). The overall fold is thus very tightly conserved across the whole population of $\mathrm{P}_{\mathrm{II}}$ proteins. The $N$. meningitidis $\mathrm{P}_{\mathrm{II}}$ T-loop tracing also indicates a similar conformation to that observed for E. coli $\mathrm{P}_{\mathrm{II}}$ and the T-loop uridylation site (Tyr51) is also conserved.

As discussed by Xu et al. (1998), analysis of the total population of available $\mathrm{P}_{\mathrm{II}}$ sequences shows that the most highly conserved residues map to the $\mathrm{P}_{\mathrm{II}}$ ATP-binding site, which is formed by the B-loop of one subunit together with the C-loop and sequences at either end of the T-loop from the adjacent subunit (Xu et al., 1998; Schwarzenbacher et al., 2004; the T-loop, B-loop and C-loop clusters are shown in Fig. 1a). As expected, the key contact residues identified by this analysis (Gly27, Thr29, Gly35, Lys58, Gly87, Gly89, Lys90, Arg101 and Arg103) are conserved in the $N$. meningitidis $\mathrm{P}_{\mathrm{II}}$ sequence and the crystal structure shows they form a pocket equivalent to that seen in E. coli (Fig. 1c), indicating that the Neisseria $\mathrm{P}_{\text {II }}$ protein is likely to bind to ATP. Analysis of mutants (Jiang, Zucker, Atkinson et al., 1997) and the mode of binding of the inhibitor 2-oxo-3-pentynoate to 4-oxalocrotonate tautomerase (Taylor et al., 1998) have also previously been combined to suggest that Gly37, Arg38, Gln39, Lys40, Thr83, Gly84, Gly89, Lys90 and Arg101 form the 2-oxoglutarate binding site (Machado Benelli et al., 2002). As can be seen in Fig. 1(a), all of these residues are conserved in the $N$. meningitidis $\mathrm{P}_{\mathrm{II}}$ sequence apart from Thr83, which has undergone a neutral mutation to Ser83, thus indicating that the Neisseria $\mathrm{P}_{\mathrm{II}}$ protein is also likely to bind 2-oxoglutarate.

In addition to these features, our analysis of multiple ClustalW alignments using sets of sequences from diverse species such as those illustrated in Fig. 1(a) has revealed a number of other patterns. Firstly, apart from residues such as Thr29 or Gly35, which are involved in both ATP binding and oligomerization, most residues forming oligomerization contacts are not strictly conserved across the whole population of $\mathrm{P}_{\text {II }}$ proteins; in part, this reflects the fact that many such contacts are formed between backbone atoms such that the nature of the side chain does not substantially affect the oligomerization. However, for the remaining contacts that do involve side-chain interactions then, as seen by Machado Benelli and coworkers in their comparison of the H. seropedicae and E. coli GlnK structures, each pair of structures compared does have some contacts in common (Machado Benelli et al., 2002). $\mathrm{P}_{\mathrm{II}}$ oligomerization-interface contacts therefore tend to show clustering of residue type, with significantly retarded genetic drift relative to the mean difference, e.g. the central oligomerization contact between Lys/Arg60 and Asp/Glu62 (Fig. 1d). This pattern suggests a strong selective pressure for maintaining the viability of normal trimer formation, which is in line with expectation as such an assembly is believed to be important for the function of $\mathrm{P}_{\mathrm{II}}$ proteins (Zhang et al., 2004). Secondly, when the residues not involved in ATP or 2-oxyglutarate binding but showing $>80 \%$ identity are mapped onto our crystal structure, they outline a hydrophobic pocket on the other side of the $\beta$-sheet to the ATP-binding site, 
together with Ile7 on the same face as the ATP site (Fig. 1d). This pattern is strongly conserved, implying that there is a functional significance to the arrangement. It is possible that the conserved residues define a binding site for an unknown ligand. Since the potential pocket is on the opposite side of the $\beta$-sheet to the ATP site, such a ligand might function as an allosteric effector regulating $\mathrm{P}_{\mathrm{II}}$ function by modulating access to the ATP-binding site. An alternative and more speculative interpretation is that the conserved residues are important in stabilizing a conformational change in the molecule. Such a change might occur during interaction with partner proteins. In this case, the hydrophobic side chains currently separated from one another and forming an open pocket might be brought into contact, forming an interdentate cross-link and making the $\mathrm{P}_{\mathrm{II}}$ protein significantly more rigid.

The interacting partners of the $\mathrm{P}_{\mathrm{II}}$ in Neisseria are currently unknown. However, given the structural conservation of the protein, some candidates can be proposed based on E. coli GlnB/GlnK. The genomes of Neisseriae encode putative UTase (e.g. NMB1203, 31\% sequence identity to E. coli) and GS-ATase enzymes (e.g. NMB0224, $35 \%$ sequence identity to $E$. coli) and we suggest that these interact with $N$. meningitidis $\mathrm{P}_{\mathrm{II}}$. The nature of the downstream effectors that functionally correspond to E. coli NRI/NRII in Neisseria spp. is less clear. The likely candidates are the co-transcribed genes that encode NtrX and NtrY (NMB0114/NMB0115), although NtrY only shows $24 \%$ sequence identity to E. coli NRII. Interestingly, the Neisseria $\mathrm{NtrY}$ protein is very similar ( $45 \%$ sequence identity) to another 2-component regulator, the atoS gene in the related species Chromobacterium violaceum. In E. coli, AtoS regulates the expression of AtoC involved in short-chain fatty-acid metabolism. In C. violaceum, which has both PII and GlnK homologues, atoS is located adjacent to $n t r X$ in the genome, reminiscent of $N$ trB/NtrC in E. coli and NtrX/ $N \operatorname{tr} Y$ in Neisseria species. Since Neisseria spp. do not appear to have separate $N \operatorname{tr} A, N \operatorname{tr} B$, atoS and atoC genes, we speculate that the single $N$. meningitidis $\mathrm{P}_{\mathrm{II}}$ may be involved in the regulation of more than one sensor system via NtrY.

The model for the regulatory role of $\mathrm{P}_{\mathrm{II}}$ is an example of direct sensing and action in which the mechanism of activation and action is dependent upon the direct modification of the sensing protein rather than upon the transcriptional control of the protein itself. Consistent with this, transcriptional profiling normally does not detect the transcript from this gene unless very high data depths $(>80 \%)$ are obtained, suggesting that it is a relatively low-abundance transcript. Furthermore, it is seldom shown to alter its expression, with the only observed changes to date being between early-log and late-log and between mid-log and late-log phase cultures, but these changes are between 1.9-fold and 1.4-fold induction and are not highly significant ( $p<0.05)$ (unpublished observations). This suggests that this gene is induced in conditions in which protein/amino-acid supply is restricted. A low constitutive expression that does not often change under differing conditions is consistent with its primary regulatory role being controlled by changes in a pre-formed protein.

The Oxford Protein Production Facility is funded by the Medical Research Council UK and is part of the Structural Proteomics in Europe (SPINE) consortium (European Commission Grant No. QLG2-CT-2002-00988).

\section{References}

Arcondeguy, T., Jack, R. \& Merrick, M. (2001). Microbiol. Mol. Biol. Rev. 65, 80-105.
Brünger, A. T., Adams, P. D., Clore, G. M., DeLano, W. L., Gros, P., GrosseKunstleve, R. W., Jiang, J.-S., Kuszewski, J., Nilges, M., Pannu, N. S., Read, R. J., Rice, L. M., Simonson, T. \& Warren, G. L. (1998). Acta Cryst. D54, 905-921.

Cheah, E., Carr, P. D., Suffolk, P. M., Vasudevan, S. G., Dixon, N. E. \& Ollis, D. L. (1994). Structure, 2, 981-990.

Chenna, R., Sugawara, H., Koike, T., Lopez, R., Gibson, T. J., Higgins, D. G. \& Thompson, J. D. (2003). Nucleic Acids Res. 31, 3497-3500.

Clamp, M., Cuff, J., Searle, S. M. \& Barton, G. J. (2004). Bioinformatics, 20, 426-427.

Collaborative Computational Project, Number 4 (1994). Acta Cryst. D50, 760-763.

Coutts, G., Thomas, G., Blakey, D. \& Merrick, M. (2002). EMBO J. 21, 536-545.

Forchhammer, K. \& Tandeau de Marsac, N. (1995). J. Bacteriol. 177, 2033 2040.

Heeswijk, W. C. van, Hoving, S., Molenaar, D., Stegeman, B., Kahn, D. \& Westerhoff, H. V. (1996). Mol. Microbiol. 21, 133-146.

Heeswijk, W. C. van, Stegeman, B., Hoving, S., Molenaar, D., Kahn, D. \& Westerhoff, H. V. (1995). FEMS Microbiol. Lett. 132, 153-157.

Heeswijk, W. C. van, Wen, D., Clancy, P., Jaggi, R., Ollis, D. L., Westerhoff, H. V. \& Vasudevan, S. G. (2000). Proc. Natl Acad. Sci. USA, 97, 39423947.

Humphrey, W., Dalke, A. \& Schulten, K. (1996). J. Mol. Graph. 14, 33-38.

Jaggi, R., van Heeswijk, W. C., Westerhoff, H. V., Ollis, D. L. \& Vasudevan, S. G. (1997). EMBO J. 16, 5562-5571.

Jaggi, R., Ybarlucea, W., Cheah, E., Carr, P. D., Edwards, K. J., Ollis, D. L. \& Vasudevan, S. G. (1996). FEBS Lett. 391, 223-228.

Jiang, P., Peliska, J. A. \& Ninfa, A. J. (1998a). Biochemistry, 37, 1278212794.

Jiang, P., Peliska, J. A. \& Ninfa, A. J. (1998b). Biochemistry, 37, 1279512801.

Jiang, P., Zucker, P., Atkinson, M. R., Kamberov, E. S., Tirasophon, W., Chandran, P., Schefke, B. R. \& Ninfa, A. J. (1997). J. Bacteriol. 179, 43424353.

Jiang, P., Zucker, P. \& Ninfa, A. J. (1997). J. Bacteriol. 179, 4354-4360.

Jones, T. A., Zou, J. Y., Cowan, S. W. \& Kjeldgaard, M. (1991). Acta Cryst. A47, 110-119.

Laskowski, R. A., MacArthur, M. W., Moss, D. S. \& Thornton, J. M. (1993). J. Appl. Cryst. 26, 283-291.

Little, R., Reyes-Ramirez, F., Zhang, Y., van Heeswijk, W. C. \& Dixon, R. (2000). EMBO J. 19, 6041-6050.

Liu, J. \& Magasanik, B. (1995). J. Bacteriol. 177, 926-931.

Machado Benelli, E., Buck, M., Polikarpov, I., Maltempi de Souza, E., Cruz, L. M. \& Pedrosa, F. O. (2002). Eur. J. Biochem. 269, 3296-3303.

Mangum, J. H., Magni, G. \& Stadtman, E. R. (1973). Arch. Biochem. Biophys. 158, 514-525.

Martinez-Argudo, I. \& Contreras, A. (2002). J. Bacteriol. 184, 3746-3748.

Ninfa, A. J. \& Atkinson, M. R. (2000). Trends Microbiol. 8, 172-179.

Ninfa, A. J. \& Jiang, P. (2005). Curr. Opin. Microbiol. 8, 168-173.

Otwinowski, Z. \& Minor, W. (1997). Methods Enzymol. 276, 307-326.

Parkhill, J. et al. (2000). Nature (London), 404, 502-506.

Ren, J., Sainsbury, S., Berrow, N. S., Alderton, D., Nettleship, J. E., Stammers, D. K., Saunders, N. J. \& Owens, R. J. (2005). BMC Struct. Biol. 5, 13.

Sakai, H., Wang, H., Takemoto-Hori, C., Kaminishi, T., Yamaguchi, H., Kamewari, Y., Terada, T., Kuramitsu, S., Shirouzu, M. \& Yokoyama, S. (2005). J. Struct. Biol. 149, 99-110.

Schwarzenbacher, R. et al. (2004). Proteins, 54, 810-813.

Taylor, A. B., Czerwinski, R. M., Johnson, W. H. Jr, Whitman, C. P. \& Hackert, M. L. (1998). Biochemistry, 37, 14692-14700.

Tettelin, H. et al. (2000). Science, 287, 1809-1815.

Thompson, J. D., Higgins, D. G. \& Gibson, T. J. (1994). Nucleic Acids Res. 22, 4673-4680.

Walter, T. S., Diprose, J. D., Brown, J., Pickford, M., Owens, R. J., Stuart, D. I. \& Harlos, K. (2003). J. Appl. Cryst. 36, 308-314.

Xu, Y., Carr, P. D., Clancy, P., Garcia-Dominguez, M., Forchhammer, K., Florencio, F., Vasudevan, S. G., Tandeau de Marsac, N. \& Ollis, D. L. (2003). Acta Cryst. D59, 2183-2190.

Xu, Y., Carr, P. D., Huber, T., Vasudevan, S. G. \& Ollis, D. L. (2001). Eur. J. Biochem. 268, 2028-2037.

Xu, Y., Cheah, E., Carr, P. D., van Heeswijk, W. C., Westerhoff, H. V., Vasudevan, S. G. \& Ollis, D. L. (1998). J. Mol. Biol. 282, 149-165.

Zhang, Y., Pohlmann, E. L. \& Roberts, G. P. (2004). Proc. Natl Acad. Sci. USA, 101, 2782-2787. 